\title{
Prediction of Thermal Decomposition Temperature of Polymers Using QSPR Methods
}

\author{
Davood Ajloo, ${ }^{*}$ Ali Sharifian, and Hossein Behniafar
}

\author{
School of Chemistry, Danghan University of Basic Science, Damghan, Iran. "E-mail: ailoo@dubs.ac.ir. \\ Received Febriary, 26, 2008
}

\begin{abstract}
The relationship between thermal decomposition temperature and structure of a new data set of eighty monomers of different polymers were studied by multiple linear regression (MLR). The stepwise nethod was used in order to variable selection. The best descriptors were selected from over 1400 descriptors including; topological, geometrical, electronic and hybrid descriptors. The effect of number of descriptors on the correlation coefficient $(R)$ and $F$-ratio were considered. Two models were suggested, one model having four descriptors $\left(R^{2}=0.894, Q_{\mathrm{cv}}^{2}=0.900, F=172.1\right)$ and other model involving 13 descriptors $\left(R^{2}=0.956, Q_{\mathrm{cv}}^{2}=\right.$ $0.956, F=125.4)$.
\end{abstract}

Key Words : Thernal decomposition temperature, Geometrical descriptors, Senti-empirical, Cross validation, Polymer

\section{Introduction}

Nowadays, before synthesizing a new material, database searching can provide information on related reactions to assist in designing viable pathways to synthesize it. In addition, computational chemistry can help to determine whether these pathways are favored from the thermodynamic point of view. Quantitative structure-property relationships (QSPR) studies can help to predict the properties of the compound of interest. The concept of QSPR is to transform searches for compounds with desired properties using chemical institution and experience into a mathematically quantified and computerized form. Once a correlation between structure and property found, any number of compounds, including those not yet synthesized, can be readily screened on computer in order to select structures with the properties desired. Thus, QSPR approach conserves resources and accelerates the process of development of new molecules for use as any purpose.

The different properties of polymer such as thermal stability, viscosity and dielectric constant, have been studied by QSPR. ${ }^{1.7}$ Thermal stability of a material is stability against degradation upon exposure to elevated temperatures in an inert environment. Polymers are often exposed to high temperatures during processing and/or use. Thus, thermal stability is among the most important properties of polymers for a wide range of applications. ${ }^{8}$ Thermal stability can be expressed with the temperature of ten percent of decom-

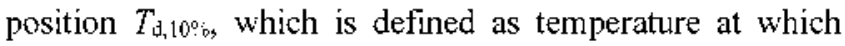
$10 \%$ loss of weight during pyrolysis was recorded by TGA at a heating rate of $10^{\circ} \mathrm{C} / \mathrm{min}$.

It can be also described with the molar thermal decomposition function $Y_{\mathrm{d}, 1: 2}$ that correlates $T_{\mathrm{d}, 12}$, with an equation: $Y_{\mathrm{d}, 1: 2}=T_{\mathrm{d}, 1: 2} \times M(M$ is the molecular weight per repeating unit). $T_{\mathrm{d}, 1: 2}$ is defined same $T_{\mathrm{d}, 10 \%}$, only for half decomposition.

Although there are numerous techniques for measuring thermal stability, it is difficulty to interpret the data and/or to compare data obtained in different laboratories or under different test condition. ${ }^{8,9}$ Some researchers have found that $Y_{d, 1: 2}$ values for polymers can be estimated on the basis of quantitative structure-property relationship models. ${ }^{1 j \cdot-13}$

One of the best-known examples of the group additive approach is that of van Krevelen. ${ }^{10}$ This method provides a rapid and computationally inexpensive approach to the estimation of $Y_{\mathrm{d}, 1: 2}$ value, but is purely empirical approach and limited to systems composed only of functional groups that have been previously investigated. Furthermore, the group contributions method is only approximate since this approach fails to account for the presence of neighboring groups or conformational influences. Bicerano extended the group additive concept for the prediction of $Y_{\mathrm{d}, 1: 2}$, based on 21 topological and constitutional descriptors. ${ }^{8}$ There are too many descriptors involved in the models though the predictions are good accuracy. In addition, Sun et al. obtained two kinds of calculated models on polymeric $Y_{d, 12}$ by artificial neural networks for linear chain polymers. " After this work, Sun et al. further built two models on polymeric $Y_{\mathrm{d}, 12}$ for the same polymeric data set by fuzzy set theory. ${ }^{12}$ The group average method is used to calculate the descriptors for two models, and the connectivity indexes method is used for them.

On the other hand, the quantum chemical descriptors used in QSPR models encode information about the electronic structure of the molecule and thus implicitly account for the cooperative effects between functional groups, charge redistribution, and possible hydrogen bonding in the polymer. ${ }^{1+}$ The two quantum chemical descriptors, obtained from the monomers of vinyl polymers were used to predict the molar thermal decomposition function $Y_{\mathrm{d}, 12.2}$. A more physically meaningful quantitative structure-property relationship (QSPR) model obtained from the training set with multiple linear stepwise regression analysis. ${ }^{7}$

The goal is to find one equation that is function of a small number of structure-based molecular descriptors that accurately predicts the experimental property. We must balance 
between increasing in number of molecular descriptors in the selected model and increasing in accuracy of it. This paper is the first time to produce a robust QSPR model that could predict $T_{0,10 \%}$ : values for polymers with different structure and family by using 1497 descriptors in 18 groups. which are calculated from the monomers of polymers by PM3 semi-empirical method. The effect of number of descriptors on statistical parameters has been analysed.

\section{Material and Methods}

Data set. One of the steps necessary for this research was the assembly of a data set. A number of structurally heterogeneous addition linear polymers were selected. The reported experimental data have been taken from Refs. [15-25]. The Thermal stability evaluation of the resulting polymers was carried out by TGA/DTG in nitrogen at a heating rate of $10^{\circ} \mathrm{C} / \mathrm{min}$. The thermograms of these polymers were almost similar with each other.

It is impossible to calculate descriptors directly for an entire molecule because all the polymers have wide distribution of molecular weight and possess high molecular weight. As we know, if the molecular weight is high enough. the terminal groups hold only a very small proportion in a polymer and its effect on the properties can be ignored. Molecular descriptors calculated directly from the structure of the repeat units can be used for the study of QSPRs for polymers, since all the properties depends on the chemical structure of the polymer molecule, and all this structure was conditioned by the repeat unit structure. Therefore, we adopt this method and focus on the following model to calculate molecular descriptors. The structures for polymers were endcapped with last group of opposing side. In the next step. the molecular structure of monomer compounds used in the polymerization of polymers, were used to determine the molecular descriptors of polymers. After providing the data set, all molecules (monomer of polymer) were drawn into Hyperchem software $e^{26}$ and optimized using the PM3 semiempirical method ${ }^{4.27}$ in Hyperchem and thereafter, topologi$\mathrm{cal}$, geometric, and electronic features of each molecule were calculated by the descriptor generation routines Dragon. ${ }^{28}$ Topological descriptors provide information about molecular shape and connectivity. Examples of these include the electrotopological state indices and molecular distance edge information geometric descriptors encode features such as shapes shadow projections, and solvent accessible surface areas. Electronic descriptors describe the electronic environment that exists within the molecule such as partial charges on each atom. There are also several hybrid descriptors, which combine several aspects of molecules molecular descriptors were calculated from the molecular structure of molecules by means of Dragon software. ${ }^{28}$

Stepwise multiple regression. In QSPR studies, the goal is to find one or more equations that are functions of a small number of structure based molecular descriptors that accurately predict the experimental property. As it is possible to generate a large number of molecular descriptors for each compound in the data set, the problem becomes how to efficiently select the set of molecular descriptors that yield an accurate relationship. If a method is sufficiently fast, it can be used to generate relationships with different numbers of molecular descriptors. The most commonly used method for this problem is the stepwise approach, which can run forward or backward.

Stepwise method was used to select the most appropriate descriptors. It is a popular technique that combines forward selection and backward elimination. It is essentially a forward selection approach, but at each step it considers the possibility of deleting a variable as in the backward elimination approach, provided that the number of model variables is greater than two. The two basic elements of the stepwise method are forward selection and backward elimination. In the forward selection the variable considered for inclusion at any step is the one yielding the largest single degree of freedom $F$-ratio among the variables that are eligible for inclusion. Consequently, at each step, the $f^{\text {th }}$ variable is added to a $k$-size model if

$$
F_{j}=\max _{j}\left(\frac{R S S_{k}-R S S_{k-j}}{s_{k-j}^{2}}\right)>F_{i n}
$$

In the above inequality RSS is the residual sum of squares and $s$ is the mean square error. The subscript $k+j$ refers to quantities computed when the $f^{\text {th }}$ variable is added to the $k$ variables that are already included in the model. In backward elimination the variable considered for elimination at any step is the one yielding the minimum single degree of freedom $F$-ratio among the variables that are included in the model. The variable is eliminated only if the corresponding $F$-ratio does not exceed a specified value $F_{\text {out }}$ Consequently, at each step, the $j^{\text {th }}$ variable is eliminated from the $k$-size model if

$$
F_{j}=\min _{j}\left(\frac{R S S_{k-j}-R S S_{k}}{s_{k}^{2}}\right)>F_{\text {OUT }}
$$

The subscript $k-j$ refers to quantities computed when the $j^{\text {th }}$ variable is eliminated from the $k$ variables that have been included in the model so far. The variable selection was done by stepwise method which implemented in SPSS software.

Cross-validation technique. The reliability of the proposed method was explored using the cross-validation method. Based on this technique, a number of modified data sets are created by deleting in each case one or a small group (leave-some-out) of objects. ${ }^{29.31}$ For each data set, an inputoutput model is developed, based on the utilized modeling technique. Each model is evaluated, by measuring its accuracy in predicting the responses of the remaining data (the ones that have not been utilized in the development of the model). In particular, the leave-one-out (LOO) procedure was utilized in this study, which produces a number of models, by deleting each time one object from the training set. Obviously, the number of models produced by the LOO procedure is equal to the number of available examples $n$. 
Prediction error sum of squares (PRESS) is a standard index to measure the accuracy of a modeling method based on the cross-validation technique. Based on the PRESS and SSY (sum of squares of deviations of the experimental values from their mean) statistics, the $Q^{2}$ and $S_{\text {PrESS values can be }}$ easily calculated. The formulae used to calculate all the aforementioned statistics are presented below (Eqs. (3) and (4)):

$$
\begin{aligned}
Q^{2}=1-\frac{P R E S S}{S S Y}=1-\frac{\sum_{i=1}^{n}\left(y_{\exp }-y_{\mathrm{pred}}\right)^{2}}{\sum_{i=1}^{n}\left(y_{\mathrm{exp}}-\bar{y}\right)^{2}} \\
S_{P R E S S}=\sqrt{\frac{P R E S S}{n}}
\end{aligned}
$$

For a more exhaustive testing of the predictive power of the model, except from the classical LOO cross-validation technique, validation of the model was carried out by a leave one-out ( $\mathrm{LOO}$ ) cross validation procedure. From the training set we randomly selected one compound. Each group was left out and that group was predicted by the model developed from the remaining observations. This procedure was carried out several times.

Estimation of the predictive ability of a QSPR model. According to Tropsha ${ }^{32}$ the predictive power of a QSPR model can be conveniently estimated by an extemal $R_{\text {cvest }}^{2}$ (Eq. (5)).

$$
R_{\mathrm{cv}, \mathrm{ext}}^{2}=1-\frac{\sum_{i=1}^{t e s t}\left(y_{\mathrm{exp}}-y_{\mathrm{pred}}\right)^{2}}{\sum_{i=1}^{t e s t}\left(y_{\mathrm{exp}}-\bar{y}_{r r}\right)^{2}}
$$

where $\bar{y}_{f r}$ is the averaged value for the dependent variable for the training set. Furthermore, the same group $\mathrm{p}^{32,33}$ considered a QSAR model predictive, if the following conditions are satisfied:

$$
\begin{gathered}
R_{\mathrm{cv}, \mathrm{ext}}^{2}>0.5 \\
R^{2}>0.5 \\
\frac{R^{2}-R_{0}^{2}}{R^{2}}<0.1 \text { or } \frac{R^{2}-R_{0}^{\prime 2}}{R^{2}}<0.1
\end{gathered}
$$

\begin{tabular}{|c|c|c|}
\hline No & Monomers used to prepare the polymers & $T_{\mathrm{d} .110 \% 0}$ \\
\hline \multicolumn{3}{|c|}{ Training set } \\
\hline 1 & $\mathrm{~N}$-[3.5-bis(N-trimellitoyl)phenyl]phthalimide and $m$-phenylenediamine & 541 \\
\hline 2 & $\mathrm{~N}$-[3,5-bis(N-trimellitoyl)phenyl]phthalimide and N-3,5-diaminophenylphthalimide & 554 \\
\hline 3 & 1.3-bis(N-trimellitoyl)benzene and $m$-phenylenediamine & 528 \\
\hline 4 & 1.3-bis(N-trimellitoyl)benzene and N-3,5-diaminophenylphthalimide & 537 \\
\hline 5 & $\mathrm{~N}$-[3,5-bis(N-trimellitoyl)phenyl]phthalimide and resorcinol & 455 \\
\hline 6 & $\mathrm{~N}$-[3,5-bis(N-trimellitoyl)phenyl]phthalimide and hydroquinone & 461 \\
\hline 7 & $\mathrm{~N}$-[3,5-bis(N-trimellitoyl)phenyl]phthalimide and 4,4'-dihydroxybiphenyl & 473 \\
\hline 8 & N-[3,5-bis(N-trimellitoyl)phenyl]phthalimide and 2,2'-dihydroxybiphenyl & 477 \\
\hline 9 & N-[3,5-bis(N-trimellitoyl)phenyl]phthalimide and 2,2'-dimethyl-4,4'-dihydroxybiphenyl & 458 \\
\hline 10 & $\mathrm{~N}$-[3,5-bis(N-trimellitoyl)phenyl]phthalimide and bisphenol-A & 451 \\
\hline 11 & $\mathrm{~N}$-[3,5-bis(N-trimellitoyl)phenyl]phthalimide and 2,7-dihydroxynaphthalene & 460 \\
\hline 12 & $\mathrm{~N}$-[3,5-bis(N-trimellitoyl)phenyl]phthalimide and 1,5-dihydroxynaphthalene & 468 \\
\hline 13 & $\mathrm{~N}$-[3,5-bis(3,4-dicarboxybenzanido phenyl]phthalimide dianhydride and $m$-phenylenediamine & 516 \\
\hline 14 & $\mathrm{~N}$-[3,5-bis(3,4-dicarboxybenzamido phenyl]phthalimide dianhydride and 2,6-diaminopyridine & 520 \\
\hline 15 & $\mathrm{~N}$-[3,5-bis(3,4-dicarboxybenzamido)phenyl]phlthalimide dianhydride and 4,4'-diaminophenylene ether & 528 \\
\hline 16 & N-[3,5-bis(3,4-dicarboxybenzanido)phenyl]phlthalimide dianhydride and $N$-(3,5-diaminophenyl)phthalimide & 528 \\
\hline 17 & N-[3,5-bis(3,4-dicarboxybenzamido)phenyl]phthalimide dianhydride and 2,2'-bis(4-aminophenoxy)-1,1'-binaphthyl & 543 \\
\hline 18 & $\mathrm{~N}$-[3,5-bis(3,4-dicarboxybenzanido)phenyl]phthalimide dianhydride and 4-p-biphenyl-2,6-bis(4-aminophenyl)pyridine & 539 \\
\hline 19 & 1,3-bis(3,4-dicarboxybenzamido)benzene dianhydride and $m$-phenylenediamine & 506 \\
\hline 20 & 1,3-bis(3,4-dicarboxybenzamido)benzene dianlyydride and 2,6-diaminopyridine & 508 \\
\hline 21 & 1,3-bis(3,4-dicarboxybenzamido)benzene diandydride and 4,4'-diaminophenylene ether & 513 \\
\hline 22 & 1.3-bis(3,4-dicarboxybenzamido)benzene dianlydride and $\mathrm{N}$-(3,5-diaminophenyl)phthalimide & 516 \\
\hline 23 & 1,3-bis(3,4-dicarboxybenzamido)benzene dianhydride and 2,2'-bis(4-aminophenoxy)-1,1'-binaphthyl & 522 \\
\hline 24 & 1,3-bis(3,4-dicarboxybenzamido)benzene diandydride and 4-p-biphenyl-2,6-bis(4-aminophenyl)pyridine & 518 \\
\hline 25 & 2,2'-bis(N-trimellitoyl)-1,1'-binaphthyl and resorcinol & 566 \\
\hline 26 & 2,2'-bis(N-trimellitoyl)-1,1'-binaphthyl and hydroquinone & 575 \\
\hline 27 & 2,2'-bis(N-trimellitoyl)-1,1'-binaphthyl and 4,4'-didydroxybiphenyl & 573 \\
\hline 28 & 2,2'-bis(N-trimellitoyl)-1,1'-bunaphthyl and 2,2'-dilydroxy-1,1'-binaphthyl & 577 \\
\hline 29 & 2.2'-bis(N-trimellitoyl)-1,1'-binaphthyl and m-phenylene diamine & 579 \\
\hline
\end{tabular}

Table 1. List of monomers of polymers* used in this research and their thermal decomposition temperature $T_{\mathrm{d}} \mid \hat{\sigma}_{*}$. 
Table 1. continued

\begin{tabular}{|c|c|c|}
\hline No & Monomers used to prepare the polymers & $T_{\text {S. } I \% \%}$ \\
\hline 30 & 2,2'-bis(N-trimellitoyl)-1,1'-binaphthyl and p-phenylene diamine & 594 \\
\hline 31 & 2,2'-bis(N-trimellitoyl)-1,1'-binaphthyll and benzidine & 596 \\
\hline 32 & 2,2'-bis(N-trimellitoyl)-1,1'-binaphthyl and 2,2'-diamino-1,1'-binaphthyl & 604 \\
\hline 33 & 4-p-biphenyl-2,6-bis(4-trimellitimidophenyl)pyridine and resorcinol & 398 \\
\hline 34 & 4-p-biphenyl-2,6-bis(4-trimellitimidophenyl)pyridine and 4,4'-dilyydroxybiphenyl & 410 \\
\hline 35 & 4-p-biphenyl-2,6-bis(4-trimellitimidophenyl)pyridine and bisphenol-A & 406 \\
\hline 36 & 4-p-biphenyl-2,6-bis(4-trimellitimidophenyl)pyridine and 2,2'-didydroxybiphenyl & 392 \\
\hline 37 & 4-p-biphenyl-2,6-bis(4-trimellitinidophenyl)pyridine and 2,2'-dilydroxy-1,1'-binaphthyl & 416 \\
\hline 38 & pyromellitic dianlyydride and $m$-phenylene diamine & 600 \\
\hline 39 & pyromellitic dianlyydride and 4,4'-diaminophenylene ether & 528 \\
\hline 40 & 3-trimellitimidobenzoic acid and $m$-phenylene diamine & 540 \\
\hline 41 & 4-p-biphenyl-2,6-bis(4-trimellitimidophenyl)pyridine and $m$-phenylene diamine & 495 \\
\hline 42 & 3.6-dimethyl-1,4-bis(methyleneisocyanate) and benzidine & 340 \\
\hline 43 & $\mathrm{~N}$-[3,5-bis(N-trimellitoyl)phenyl]phthalimide and N-3.5-Diaminophenylphthalimide & 554 \\
\hline 44 & $\mathrm{~N}$-[3.5-bis(N-trimellitoyl)phenyl]phthalimide and $m$-phenylene diamine & 541 \\
\hline 45 & $\mathrm{~N}$-[3,5-bis(N-trimellitoyl)phenyl]phthalinide and resorcinol & 455 \\
\hline 46 & 2,2'-bis(4-trumellitumidophenoxy)biphenyl and $m$-phenylene diamine & 507 \\
\hline 47 & 2,2'-bis(4-trimellitimidophenoxy)biphenyl and 2,6-diaminopyridine & 511 \\
\hline 48 & 2,2'-bis(4-trumellitimidophenoxy)biphenyl and $p$-phenylene diamine & 520 \\
\hline 49 & 2,2'-bis(4-trinellitinidophenoxy)biphenyl and 4,4'-diaminophenylene ether & 531 \\
\hline 50 & 2,2'-bis(4-trimellitumidophenoxy)biphenyl and 4-phenyl-2,6-bis(4-aminophenyl)pyridine & 545 \\
\hline \multicolumn{3}{|c|}{$\begin{array}{ll}\text { Testing set } \\
\end{array}$} \\
\hline 51 & 2,2'-bis(4-trimellitimidophenoxy)-1,1'-binaphthyl and $m$-phenylene diamine & 512 \\
\hline 52 & 2,2'-bis(4-trimellitimidophenoxy)-1,1'-binaphthyl and 2,6-diaminopyridine & 517 \\
\hline 53 & 2,2'-bis(4-trimellitimidophenoxy)-1,1'-binaphthyl and $p$-phenylene diamine & 524 \\
\hline 54 & 2,2'-bis(4-trimellitimidophenoxy)-1,1'-binaphthyl and 4,4'-diaminophenylene ether & 535 \\
\hline 55 & 4-phenyl-2,6-bis(4-trimellitimidophenyl)pyridine and $m$-phenylene diamine & 485 \\
\hline 56 & 4-phenyl-2,6-bis(4-trimellitimidophenyl)pyridine and 2,6-diaminopyridine & 502 \\
\hline 57 & 4-phenyl-2,6-bis(4-trimellitimidophenyl)pyridine and 4-phenyl-2,6-bis(4-aminophenyl)pyridine & 516 \\
\hline 58 & 4-p-biphenyl-2,6-bis(4-trimellitimidophenyl)pyridine and $m$-phenylene diamine & 485 \\
\hline 59 & 4-p-biphenyl-2,6-bis(4-trimellitimidophenyl)pyridine and 2,6-diaminopyridine & 510 \\
\hline 60 & 2,2'-bis(4-trimellitimidophenoxy)biphenyl and resorcinol & 453 \\
\hline 61 & 2,2'-bis(4-trimellitimidophenoxy)biphenyl and hydroquinone & 467 \\
\hline 62 & 2,2'-bis(4-trimellitimidophenoxy)biphenyl and 2.2'-dihydroxybiphenyl & 458 \\
\hline 63 & 2,2'-bis(4-trimellitimidophenoxy)biphenyl and 4,4'-dihydroxybiphenyl & 470 \\
\hline 64 & 2,2'-bis(4-trimellitimidophenoxy)biphenyl and bisphenol-A & 449 \\
\hline 65 & 2,2'-bis(4-trimellitimidophenoxy)biphenyl and 2,2'-dihydroxy-1,1'-binaphthyl & 484 \\
\hline 66 & 4-p-biphenyl-2,6-bis(4-trimellitimidophenyl)pyridine and resorcinol & 448 \\
\hline 67 & 4-p-biphenyl-2,6-bis(4-trimellitimidophenyl)pyridine and hydroquinone & 452 \\
\hline 68 & 4-p-biphenyl-2,6-bis(4-trimellitimidophenyl)pyridine and 4,4'-dilydroxybiphenyl & 464 \\
\hline 69 & 4-p-biphenyl-2,6-bis(4-trimellitimidophenyl)pyridine and 2,2'-dilydroxybiphenyl & 449 \\
\hline 70 & 4-p-biphenyl-2,6-bis(4-trimellitimidophenyl)pyridine and 2,2'-dimethyl-4,4'-dihydroxybiphenyl & 441 \\
\hline 71 & 4-p-biphenyl-2,6-bis(4-trimellitimidophenyl)pyridine and bisphenol- $\mathrm{A}$ & 433 \\
\hline 72 & 4-p-biphenyl-2,6-bis(4-trimellitimidophenyl)pyridine and 2,7-dihydroxynaphthalene & 459 \\
\hline 73 & 4-p-biphenyl-2,6-bis(4-trimellitimidophenyl)pyridine and 1,5-dibydroxynaphthalene & 455 \\
\hline 74 & 4-p-biphenyl-2,6-bis(4-trimellitimidophenyl)pyridine and 2,2'-dilydroxy-1,1'-binaphthyl & 471 \\
\hline 75 & $\mathrm{~N}-[3,5$-bis(N-trimellitoyl)phenyl]phthalimide and resorcinol & 391 \\
\hline 76 & $\mathrm{~N}-[3,5$-bis(N-trimellitoyl)phenyl]phthalimide and hydroquinone & 399 \\
\hline 77 & $\mathrm{~N}-[3,5$-bis(N-trimellitoyl)phenyl]phthalimide and 4,4'-dihydroxybiphenyl & 404 \\
\hline 78 & $\mathrm{~N}$-[3,5-bis(N-trimellitoyl)phenyl]phthalimide and 2.2'-dilhydroxybiphenyl & 403 \\
\hline 79 & $\mathrm{~N}-[3,5-b i s(\mathrm{~N}$-trimellitoyl)phenyl]phthalimide and bisphenol-A & 385 \\
\hline 80 & $\mathrm{~N}$-[3,5-bis(N-trimellitoyl)phenyl]phthalimide and 2.2'-dihydroxy-1,1'-biuaphthyl & 412 \\
\hline
\end{tabular}

*Data were taken from references $15-25$ 


$$
0.85 \leq k \leq 1.15 \text { or } 0.85 \leq k^{\prime} \leq 1.15
$$

Mathematical definitions of $R_{0}^{2}, R_{0}^{\prime 2}, k$ and $k^{\prime}$ are based on regression of the observed activities against predicted activities and vice versa (regression of the predicted activities against observed activities). The definitions are presented clearly in Golbraikh et al ${ }^{33}$

\section{Results and Discussion}

Table 1 shows data set for 80 different groups including poly(amide-imide), poly(ester-imide) and poly(urethaneimide) of polymers and their thermal decomposition temperature which are taken from Refs. [15-25].

The data of $T_{\mathrm{d}, 10^{\circ} \mathrm{o}}$ in Table 1 are divided into a training set and a validation set. The former includes 50 polymeric (150) $T_{d, 10 \%}$ values; while the latter with 30 polymers $(51-80)$ data are obtained from the experimental data of $T_{0,110 \%}$. Training set is used to build a QSPR model, which is evaluated with the validation set. It is impossible to calculate descriptors directly for an entire molecule because all polymers have wide distribution of molecular weights and possess high molecular weights. Molecular descriptors calculated directly from the structure of the monomers can be used on the study of QSPR models for polymers, since all the properties depend on the chemical structure of the polymer molecule, and all this structure is conditioned by the monomer structure.

Stepwise method selects the descriptors step-by-step. The best 13 selected descriptors among 1497 descriptors were tabulated in Table 2. They are belongs to different class of descriptors such as autocorrelation, 3D-MoRSE, topological, shape and aromaticity. ${ }^{28}$

Molecular descriptors based on the autocorrelation function $A C_{\text {, }}$, defined as:

$$
A C_{l}=\int_{a}^{b} f(x) \cdot f(x+1) \cdot d x
$$

Where $f(x)$ is any function of variable $x$ and $l$ is the lag representing an interval of $x ; a$ and $b$ defined the total studied interval of the function. The function $f(x)$ is usually time-dependent function such as a time dependent electrical signal, or a spatial-dependent function such as the population density in space. Function $A C_{l}$ is a summation of the function value products calculated at $x$ and $x+l$. To obtain spatial autocorrelation molecular descriptors, function $f(x)$ is any physico-chemical property calculated for each molecule, such as atomic mass, polarizability, etc. Therefore, the molecule atom represents the set of discrete points in space and the atomic property evaluated at those points. $A C$ descriptors can also be calculated for $2 \mathrm{D}$ and $3 \mathrm{D}$-spatial molecular geometry. In $2 \mathrm{D}$ case the distribution of a molecular property can be a mathematical function $f(x, y)$ for each point. The most well known autocorrelation descriptors are a) Moran coefficient $(I(d))$ and b) Geary coefficient $c(d)$.

General index of spatial Moarn autocorrelation that, if applied to a molecular graph, can be defined as:

$$
I(d)=\frac{\frac{1}{\Delta} \sum_{i=1}^{A} \sum_{j=1}^{A} \delta_{i j} \cdot\left(w_{j}-\bar{w}\right)\left(w_{j}-\bar{w}\right)}{\frac{1}{A} \cdot \sum_{i=1}^{A}\left(w_{j}-\bar{w}\right)^{2}}
$$

In addition Geary descriptors are defined as:

$$
c(d)=\frac{\frac{1}{2 \Delta} \sum_{i=1}^{A} \sum_{j=1}^{A} \delta_{i j}\left(w_{i}-w_{2}\right)^{2}}{\frac{1}{(A-1)} \cdot \sum_{i=1}^{A}\left(w_{i}-\bar{w}\right)^{2}}
$$

Where $w_{i}$ is any atomic property, $\bar{w}$ is its average value on the molecule. $A$ is the atom number, $d$ is the considered topological distance (i.e. the lag in autocorrelation terms), $\delta_{\mathrm{ji}}$ is a Kronecker delta ( $\delta_{\mathrm{j} j}=1$ if $d_{\mathrm{jij}}=d$, zero otherwise), $\Delta$ is the sum of the Kronecker deltas, i.e. the number of vertex pairs at distance equal to $d^{28}$ The Moran coefficient usually

\begin{tabular}{|c|c|c|}
\hline Symbol & Definition & Class \\
\hline MATS2e & Moran autoconrelation - $\operatorname{lag} 2 /$ weighted by atomic Sanderson electronegativities & 2D autocorrelations \\
\hline GATS1v & Geary autocorrelation - lag 1 / weighted by atomic van der Waals volumes & $2 \mathrm{D}$ autocorrelations \\
\hline GATS8e & Geary autocorrelation $-\operatorname{lag} 8 /$ weighted by atomic Sanderson electronegativities & 2D autocorrelations \\
\hline HATS $5 v$ & leverage-weighted autoconelation of lag 5 weighted by atomic van der Waals volumes & GETAWAY descriptors \\
\hline MATS8p & Moran autocorrelation $-\operatorname{lag} 8$ : weighted by atomic polarizabilities & $2 \mathrm{D}$ autocorrelations \\
\hline Mor28u & 3D-MoRSE - signal 28 : unweighted & 3D-MoRSE descriptors \\
\hline $\mathrm{BEHm} 2$ & highest eigenvalue n. 2 of Burden matrix / weighted by atomic masses & BCUT descriptors 132 \\
\hline DECC & Eccentric & topological descriptors \\
\hline AROM & aromaticity (trial) & aromaticity indices \\
\hline Mor19p & 3D-MoRSE - signal 19 / weighted by atomic polarizabilities & 3D-MoRSE descriptors \\
\hline PJI2 & 2D Petitiean shape index & topological descriptors \\
\hline Mor29e & 3D-MoRSE - signal 29 - weighted by atomic Sanderson electronegativities & 3D-MoRSE descriptors \\
\hline Mor27m & 3D-MoRSE - signal 27 - weighted by atomic masses & 3D-MoRSE descriptors \\
\hline
\end{tabular}
takes a values in the interval $[-1,+1]$. Positive autocorrelation corresponds to positive values of the coefficient

Table 2. Selected descriptors by stepwise nuthod 
whereas negative autocorrelation produces negative values.

The Geary coefficient, $c(d)^{28}$ is a distance type function varying from zero to infinity. Strong autocorrelation produces low values of this index; moreover, positive autocorrelation translates into values between 0 , and 1 whereas negative autocorrelation produce values larger than 1 ; therefore the reference "no correlation" is $c=1$.

3D-MoRSE descriptors (3D-Molecule Representation of Structure based on Electron diffaction) are based on the idea of obtaining information from the $3 \mathrm{D}$ atomic coordinates by the transform used in electron diffraction studies for preparing the practical scattering curves. ${ }^{28} \mathrm{~A}$ generalized scattering function called the molecular transform, can be used as the functional basis for deriving. from a known molecular structure, the specific analytic relationship at both $\mathrm{X}$-ray and electron diffraction.

Molecular eccentricity $(\varepsilon)$ : Among the eigenvalue-based descriptors, molecular eccentricity is a shape descriptor obtained from the eigenvalues, $\lambda$, of the inertia matrix $I$ defined as follow ${ }^{28}$ :

$$
\varepsilon=\frac{\left(\lambda_{1}^{2}-\lambda_{3}^{2}\right)^{1 / 2}}{\lambda_{1}} \quad 0 \leq \varepsilon \leq 1
$$

Where $\varepsilon=0$ corresponds to spherical top molecules and $\varepsilon=$ 1 to linear molecules.

It is a shape descriptor defined by analogy with the eccentricity of ellipse, which is defined:

$$
\varepsilon=\frac{\left(l_{M}^{2}-l_{m}^{2}\right)^{1 / 2}}{l_{M}}
$$

Where $l_{\mathrm{M}}$ and $l_{\mathrm{m}}$ are the lengths of the major and minor elliptical axes, respectively.

BCUT descriptors: defined as eigenvalues of a modified connectivity matrix, which could be called Burden matrix $B .^{28}$ The $B$ matrix is defined as the following: The diagonal elements $B_{\mathrm{ij}}$ are the atomic number $Z_{i}$ of the atoms; the offdiagonal elements $B_{\mathrm{ij}}$ representing two bonded atoms $i$ and $j$ are equal to $\pi^{*} \cdot 10^{-1}$ where $\pi^{*}$ is conventional bond order, i.e. $0.1,0.2,0.3$ and 0.15 for single, double, triple and aromatic bond respectively; off-diagonal elements $B_{\mathrm{ij}}$ corresponding to terminal bonds are augmented by 0.01 ; all other matrix elements are set a 0.001 .

One aim of this work is also studying the effect of number of descriptors on the some statistical parameters such as correlation coefficient $(R)$ and $F$ ratio $(F)$. The stepwise method in SPSS automatically selects descriptors. In each step, one descriptor is added. In this method 13 descriptors were selected step by step. Each step usually named as a model. Figure 1 shows the effect of number of descriptors on the $R$ and $F$ variations. Increasing the number of descriptors increases the $R$ and $F$ up to four descriptors. After that, we see increment of $R$ and decrement of $F$ up to thirteenth descriptor. It must be noted that after inclusion of fourth descriptor the variation of $R$ is not significant (the curve is smooth and has lower slope). Thus we have two regions, one before fourth descriptors another after it. We named for

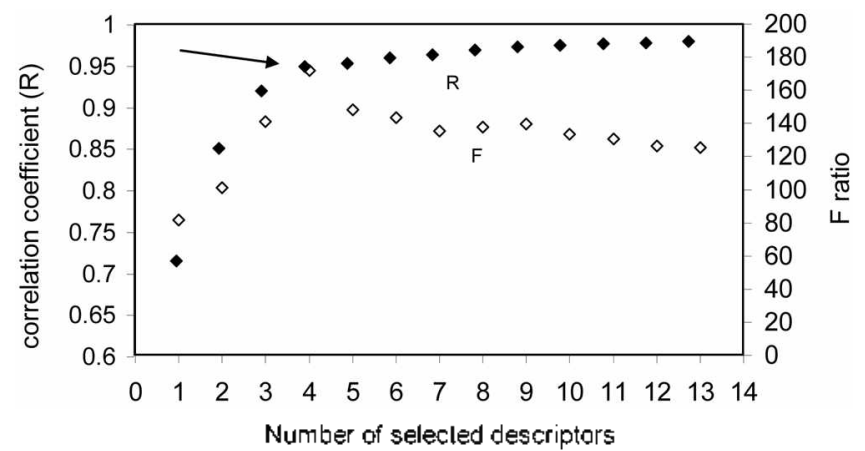

Figure 1. Effect of number of descriptors on the correlation coefficient $(R)$ and $F$-ratio.

simplicity the model which having four descriptors as model 1 and model which having thirteen descriptors as model 2. Before model 1 we have lower $R$ after that we have lower $F$. Thus model 1 which has highest $F$ and lowest number of descriptors and regional $R$ is the best model.

The effect of number of descriptors on the correlation coefficient $(R)$ and $F$-ratio $(F)$ shown in Figure 1 . It is observed that $R$ is increased due to increasing the number of descriptors. But, $F$-ratio increases up to descriptor 4 and then be decays downward to descriptor 13 . The optimum model is that has higher $R, F$ and smaller number of descriptors. Thus, in this work we selected two models, one model including four descriptors (model 1) and other including thirteen descriptors (model 2 ).

By carrying out the correlation between the molar thermal

Table 3. Coefficient of each descriptor and related error associated with their mean effects

\begin{tabular}{lrrrr}
\hline \multicolumn{5}{c}{ Model 1 } \\
\hline & coefficient & Std. Error & Mean effect & Sig. \\
(Constant) & 1314.393 & 67.220 & & $3.6 \mathrm{E}-31$ \\
MATS2e & -460.310 & 45.976 & -0.417 & $1.81 \mathrm{E}-15$ \\
GATS1v & -1061.106 & 83.345 & -0.505 & $1.99 \mathrm{E}-20$ \\
GATS8e & 115.397 & 13.055 & 0.323 & $3.02 \mathrm{E}-13$ \\
HATS5v & 988.222 & 154.175 & 0.247 & $1.16 \mathrm{E}-08$ \\
\multicolumn{5}{c}{ Model 2 } \\
\hline & coefficient & Std. Error & Mean effect & Sig. \\
(Constant) & 4408.825 & 523.903 & & $4.79 \mathrm{E}-12$ \\
MATS2e & -421.865 & 40.779 & -0.382 & $1.9 \mathrm{E}-15$ \\
GATS1v & -1070.665 & 91.227 & -0.510 & $8.28 \mathrm{E}-18$ \\
GATS8e & 131.712 & 15.508 & 0.369 & $3.48 \mathrm{E}-12$ \\
HATS5v & 495.247 & 148.470 & 0.124 & 0.0014 \\
MATS8p & -245.815 & 66.738 & -0.167 & 0.000466 \\
Mor28u & 30.794 & 5.329 & 0.209 & $2.23 \mathrm{E}-07$ \\
BEHm2 & -502.815 & 75.351 & -0.310 & $6.15 \mathrm{E}-09$ \\
DECC & 17.355 & 4.058 & 0.242 & $6.26 \mathrm{E}-05$ \\
AROM & -1045.937 & 260.139 & -0.202 & 0.000152 \\
Mor19p & -18.892 & 6.882 & -0.100 & 0.007784 \\
PJI2 & -136.303 & 49.755 & -0.070 & 0.007907 \\
Mor29e & -14.730 & 5.978 & -0.101 & 0.016354 \\
Mor27m & -16.625 & 6.917 & -0.112 & 0.019074 \\
\hline & \multicolumn{5}{c}{}
\end{tabular}




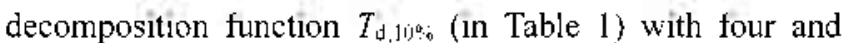
thirteen descriptors using stepwise regression analysis, two models obtained (Table 3). Table 3 includes coefficients of two equations for both models. Coefficient of each descriptor. related mean effect and standard error for them are listed in the table. Negative coefficient shows the reverse trend of that descriptor and thermal decomposition temperature. Mean effect represents the effect of each descriptors on $T_{\mathrm{d}, 1 \mathrm{i} \mathrm{j}_{0}}$

Present version of Dragon software calculates 1497 descriptors in 18 different classes belong to atomic constitution, atomic correlation, molecular topology, steric properties and so on. All of them are physico-chemical properties. The selected physico-chemical properties meaning defined in the above by formula. But they may be not popular as well as molecular weight, volume, polarity and.... Correlation between $T_{\mathrm{d}, 10 \%}$ and these selected descriptors were taken in Table 3. We can write it as following equation for model 1 :

$T_{t^{\prime}, 10 \%}=-460.310($ MATS2e $)-1061.106($ GATS1 $\nu)$

$$
\text { + 115.397(GATS8e })+988.222(\text { HATS5 } v)+1314.393
$$

These four variables were briefly defined in the Table 2 and discussed with more detailed in the above. Negative and
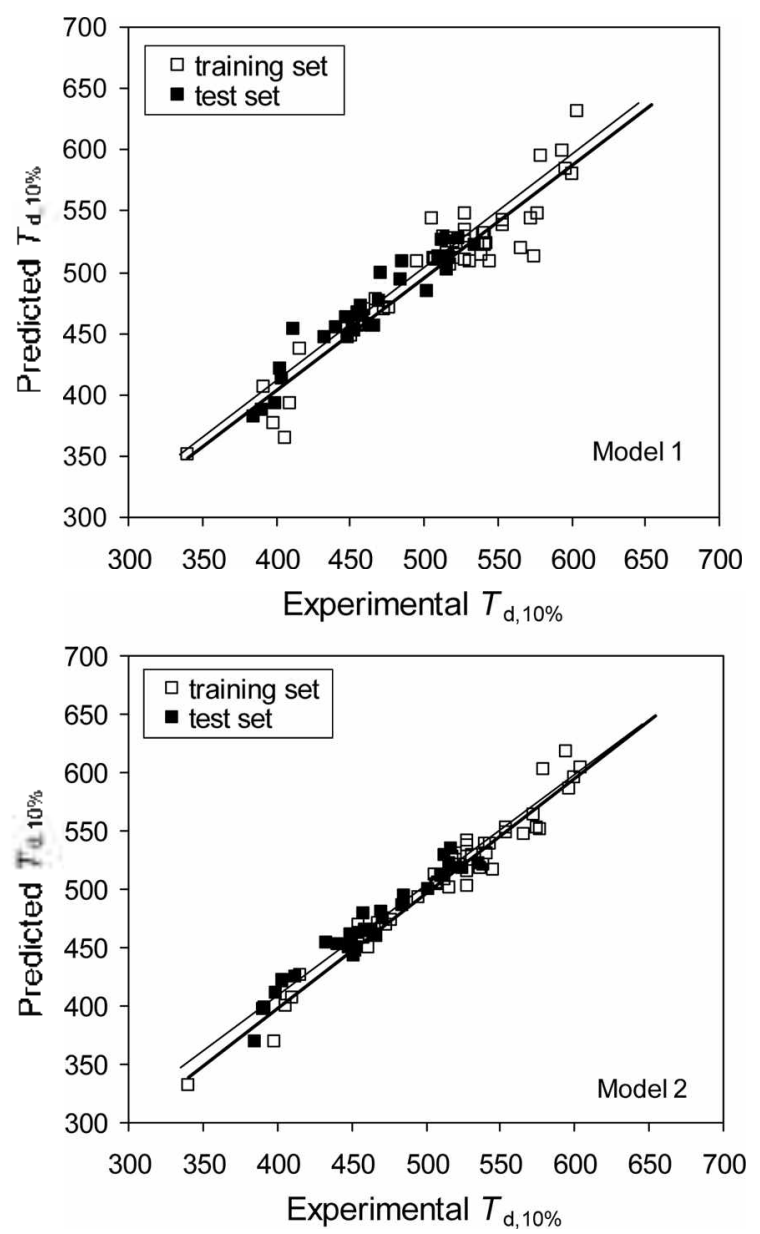

Figure 2. Predicted versus experimental thermal decomposition temperature $\left(T_{\mathrm{i}, 1}\left(x_{0}\right)\right.$ ) for two models; (a) model l including four descriptors and (b) model 2 including thirteen descriptors. Narrow and bold tredlines are related to testing and training set: respectively. positive coefficients make decreases and increases $T_{\mathrm{d}, 1(10: ;}$, respectively. $T_{\mathrm{d}, 1110:}$ and more customary descriptors have weak correlation, so that they have not been selected in here. Also some of reports in the literature used descriptors which are similar to our descriptors and were not discussed detailed definition of descriptors. ${ }^{34}$

Variables in the model 1 and 2, are topological or geometrical. Variables in model 1 are two dimensional descriptors, namely they depend on two variable coordinates such as $\mathrm{x}$ and $\mathrm{y}$ or $\mathrm{x}$ and $\mathrm{z}$ or $\mathrm{y}$ and $\mathrm{z}$. While variables in model 2 are two and three dimensional.

Two dimensional properties may be due to surface dependent properties, such as aromaticity. On the other hand, some part of our sample molecules have aromatic nature. Thus, significantly appearance of two dimensional properties may be due necessity for coplanarity.

The predicted versus experimental $T_{\mathrm{d}, l\left[\left[_{0}, \mathrm{i}\right.\right.}$ values for model 1 and 2 are shown in Figure 2. It is clearly represented that both model are satisfactory and model 2 has slightly higher correlation coefficient relative to model 1 . But model 1 has lower higher $F$ and lower number of descriptors. Thus model 1 is the better model.

The Leave-One-Out (LOO) cross-validation method is adopted to test the internally predictive ability for Eq. (3) for two models. The cross-validation $Q^{2}$ crim value for medel 1 is 0.885 . Then the model is evaluated extemally using the test set of 30 polymers (in Table 1). The cross-validation $Q^{2}$ cvest value for the external test set is 0.884 . Coefficients of determination $R_{0}^{2}$ (predicted versus observed $T_{\mathrm{d}, 10^{\circ} \text { i }}$ values) and $R_{\|}$(observed versus predicted $T_{\mathrm{d}, 1(10 \% ;}$ values) are 0.885 and 0.899 , respectively. The slopes of regression lines through the origin of predicted versus observed $k$ and observed versus predicted $k^{\prime}$ values are 1.013 and 0.986 , respectively. According to Refs. [35-36], a QSPR model is successful if it satisfied several criteria as follows: (1) $Q^{2}$ wrint $>0.5, Q_{\text {cr.ext }}^{2}>0.5, R^{2}>0.6 ;(2) R_{0}^{2}$ and $R_{0}^{\prime 2}$ close to $R^{2},\left(R^{2}\right.$

Table 4. Statistical parameters for two models

\begin{tabular}{|c|c|c|}
\hline Statistics & Model 1 & Model 2 \\
\hline No. of descriptors & 4 & 13 \\
\hline$R^{\prime}$ & 0.894 & 0.956 \\
\hline$F$ & 172.1 & 125.4 \\
\hline$N$ & 80 & 80 \\
\hline Sig & $5.66 \mathrm{E}-37$ & $3.1+\mathrm{E}-41$ \\
\hline Std. Enror & 18.6 & 12.5 \\
\hline$Q^{\prime}$ & 0.900 & 0.956 \\
\hline$Q_{c t, \mathrm{jot}}$ & 0.886 & 0.956 \\
\hline$Q_{\text {cr,ext }}$ & 0.884 & 0.931 \\
\hline$R_{0}^{2}$ & 0.893 & 0.941 \\
\hline$\left(R^{2}-R_{1},{ }^{2}\right) / R^{2}$ & 0.001 & 0.016 \\
\hline$\left.R_{0}^{\prime}\right)^{2}$ & 0.893 & 0.941 \\
\hline$\left(R^{2}-R^{\prime} 0^{3}\right) / R^{2}$ & 0.001 & 0.016 \\
\hline$k$ & 1.013 & 1.011 \\
\hline$R_{\text {creat }}$ & 0.938 & 0.965 \\
\hline$k^{\prime}$ & 0.986 & 0.988 \\
\hline
\end{tabular}


$\left.-R_{0}^{2}\right) / R^{2}<1,\left(R^{2}-R_{0}^{2} y / R^{2}<0.1(3) k\right.$ and $k^{\prime}$ are between 0.85 and 1.15 . The cited statistical parameters for models 1 and 2 were listed in Table 4 . Thus, in model 1

$$
\begin{aligned}
& T_{\mathrm{d}, 10 \%}(\text { pred })=0.925 T_{\mathrm{d}, 10^{\circ} \circ}(\exp )+41.04 n=80 \mathrm{~F}=172 \\
& S=18.6 \quad R^{2}=0.894
\end{aligned}
$$

And in model 2

$$
\begin{aligned}
& T_{\mathrm{d}, 10 \%}(\text { pred })=0.950 T_{\mathrm{d}, 10 \%}(\exp )+28.39 n=80 \mathrm{~F}=125 \\
& S=12.5 R^{2}=0.956
\end{aligned}
$$

It is obvious that our results satisfy the generally accept condition. Therefore, the QSPR model obtained in this paper is suitable for making accurate prediction outside of the training set of polymers. According to the Sig.-test (see Table 2), the descriptors appearing in correlation equations are significant descriptors. The standard errors of estimation in Eq. (5) for model 1 and 2 are $18.6 \mathrm{~K}$ and 12.5 , respectively.

Finally, an overview on the total results, shows that. increasing the number of descriptors increases the $R$, but $F$ up to fourth descriptors then decreases after that descriptor. Thus, model 1 is optimum model which have lower number of descriptors and higher $F$ and acceptable $R$

\section{Conclusion}

The QSPR is a method in order to prediction of polymer properties. In this work, a number of descriptors have been selected among a large number of descriptors. The selected descriptors were belongs to different class of descriptors such as: topological, electronic, geometrical and hybrid descriptors. The effect of number of descriptors also was addressed. On the basis of this effect, two models were presented. The model which having less number of descriptors. has lower correlation coefficient, lower cross validation and higher $F$-ratio. The optimum model is one which has higher $R$, higher $F$ and lower number of descriptors. Thus, It seems those model which having four descriptors is optimum and better model.

Acknowledgments. Financial support of Damghan University of Basic Science and very useful comments from Dr. Bahram Hemmtinejad are acknowledged.

\section{References}

1. Mattioni, B. E.; Turs, P. C. J. Chent Inf. Comput. Sei. 2002, 42, 232

2. Liu, A.; Wang, X.; Wang, L.; Wang, H.; Wang, H. Em: Polvm. $J$. $\mathbf{2 0 0 7}, 43,989$

3. Afantitis, A.; Melagraki, G.; Sarimveis, H.; Koutentis, P. A.; Markopoulos, I.; Igglessi-Markopoulou, O. Polvmer 2006, 47, 3240.

4. Gharagheizi, F. Comp. Mater Sci. 2007, 40, 159.

5. Xua, J.; Chenb, B.; Zheng, Q.; Guob, B. Polymer 2004, 4.5, 8651 .

6. Yu, X.; Yi, B.; Xie, Z.; Wang, X.; Liu, F. Chenomen: Inell. Lab. $2007,87,247$

7. Yu, X.; Xie, Z.; Yi, B.; Wang, X.; Liu, F. Eur: Polvm. J. 2007, 43, 818.

8. Bicerano, J. Prediction of Polymer Properties. 2nd ed.; Marcelar Dekker Inc.: New York, 1996.

9. Flymu, J. H. I. Therm, Anal. 1988, 34, 367.

10. van Krevelen, D. W. Properties of Polymers. 3rd ed.; Elsevier: Amsterdam, 1990.

11. Sun, H.; Tang, Y. W.; Wu, G. S.; Zhang, F. S.; Chen, X. Q. Comput. Appl. Chem. 2003, 20, 210.

12. Sun, H.: Tang, Y. W.; Wu, G. S.; Zhang, F. S.; Chen, X. Q. Comp. Appl. Chem. 2003, 20,381.

13. Yu, X.; Xie, Z.; Yi, B.; Wang, X.; Liu, F. Em: Polm. J. 2007, 43, 818.

14. Katritzky, A. R.; Sild, S.; Karelson, M. J. Chem. Inf. Comput. Sci. $1998, \delta, 1171$

15. Behniafar, H.; Haghighat, S. Eur: Polvm. J. 2006, 42, 3236.

16. Behuiafar, H.; Akhlaghinia, B.; Habibian, S. Eum Polvm. J. 2005, 41,1071 .

17. Behuiafar, H. J. Appl. Polm. Scr. 2006, 101, 869 .

18. Behniafar, H.; Khaje-Mirzai, A. A.; Beit-Saeed, A. Polmm. Int. $\mathbf{2 0 0 7}, 56,74$.

19. Banihashemi, A.; Behuiafar, H. Polvm. Int. 2003, 52, 1136.

20. Behuiafar, H.; Habibian, S. Polm. Int. 2005, 54, 1134.

21. Behniafar, H.; Banihashemi, A. Eur: Polm w. J. 2004, 40, 1409.

22. Behniafar, H.; Jafari, A. J. Appl. Polym. Sci. 2006, 100, 3203.

23. Behniatar, H. J. Macromol. Sci. Pur. Appl. Chem. 2006, $43,813$.

24. Behniafar, H.; Haghighat, S.; Farzanel, S. Polymer 2005, 46 , 4627.

25. Behuiafar, H.; Banihashemi, A. Polym. In. 2004, 53, 2020.

26. Motamedi, M.; Bathaje, S. Z.; Hemmateenejad, B.; Ajloo, D. J. Mol. Struchure Theochemi 2004, 678, 163.

27. HiperChem Release 7.5 for Windows, Molecular Modeling System; Hypercube, lnc: 2002.

28. (a) Todechini, R.; Consoni, V. Handbook of Molecular Descriptors; Wiley VCH: 2000. (b) Talete srl, Dragon for Windons (Software for molecular Descriptor Calculations), Version 5,4-2006.

29. Efron, B. J. Ant. Stat. Assoc. 1983, $78,316$.

30. Efroymson, М. A. Mitultiple Regression Analysis in Mathematical Wethods for Digital Computers; Ralston, A., Wilf, H. S., Eds.; Wiley; New York, 1960.

31. Osten, D. W.J. Chemom. 1998, 2, 39.

32. Tropsha, A.; Gramatica, P.; Gombar, V. K. Quant. Shruct. Activ: Relat. 2003, 22, 1.

33. Golbraikh, A.; Tropsha, A. J. Mol. Graphics Model 2002, $20,269$.

34. Brian, E. M.: Peter, C. J. J. Chem. Inf. Comput. Sci. 2002, 42, 232.

35. Efron, B. J. An. Stat. Assoc. 1983, 78, 316.

36. Efroymson, M. A. Mithiple Regression Anolusis in Mathenatical Methods for Digital Computers; Ralston, A., Wilf, H. S., Eds.; Wiley: New York, 1960. 